\title{
Study on Thickness Calculation Method for Undigraph
}

\author{
Yan $\mathrm{Cui}^{\mathrm{a},{ }^{*} 1}$, Chao-Dong Cui ${ }^{\mathrm{b},{ }^{* 2}}$
}
${ }^{a}$ Computer Science and Engineering Department, North China Institute of Aerospace Engineering, Langfang, 065000, China
${ }^{b}$ China Academy of Building Research, Beijing, 100013, China

\begin{abstract}
How to solve the thickness of general graph is an unsolved problem and one of many difficult problems in graph theory. This paper studies the method for calculating the thickness of undigraph and proposes formula and concrete method for calculating the thickness of undigraph G. Meanwhile, method is proposed for testing whether division of side-derived subgraphs is correct. Some instances presented in this paper aims at illustrating the actual application and correctness of calculation of this method for reference.
\end{abstract}

Index Terms: Thickness of Graph; Side-Derived Subgraph; Maximal Planar Graph;Nonplanar Graph; Euler's Planar Graph Formula

(C) 2011 Published by MECS Publisher. Selection and/or peer review under responsibility of the Research Association of Modern Education and Computer Science

\section{Definition of Thickness of Graph $G$}

For given undigraph $G=<V, E>$, it is called a planar graph if all nodes and sides of it can be drawn on a plane and all its sides do not interconnect each other except nodes. Also, the drawn graph is called a planar embedding of graph $G$. Graph $G$ is called a non-planar graph if some sides invariably interconnect each other besides nodes no matter how many redraws are done to $G$.

Suppose that $G=<V, E>$ is a non-planar graph, according to the definition, there must be some sides invariably interconnecting each other. Then, how to embed the sides of this graph is obviously a problem worthy to be studied.

Obviously, the solution for solving planar embedding problem of interconnecting sides (except for nodes) of non-planar graph $G$ is to embed its sides into n planes to make sides in different plan without interconnection.

So, let's divide the side set of $G$ and make division satisfy the condition: $E(G)=\bigcup_{i=1}^{n} E_{i}, E_{i} \cap E_{j}=\phi$ $i \neq j$ and all side-derived subgraphs $G\left(E_{i}\right)(i=1,2, \cdots, n)$ are planar graphs.

Then, the minimum value of $\mathrm{n}$ is the thickness $\theta(G)$ of graph $G$.

* Corresponding author:

| E-mail address: ${ }^{* 1}$ lfhtcy3238@ sina.com; ${ }^{* 2}$ lfd1972@ sohu.com 


\section{Initiation of Question}

According to the definition of thickness of graph $G$, it is obvious that the thickness of planar graph is 1 , whereas the minimum thickness of non-planar graph is 2 . However, the problem about thickness of general graph is still unsolved. For this issue, Professor Wang Shuhe, mathematician of our country said: "thickness calculation of general graph is an unsolved problem. By now, neither formula nor effective algorithm for thickness calculation is not still proposed and established."

- $\theta(G) \geq\left\{\frac{E}{3 V-6}\right\}, V>2,\{x\}$ is integer part of x plus 1 ;

- if there is no third order loop in the connected graph $G$, then $\theta(G) \geq\left\{\frac{E}{2 V-6}\right\}, V>2$;

- $\theta\left(K_{v}\right) \geq\left\{\frac{V+7}{6}\right\}, V \geq 3,\{x\}$ is integer part of $\mathrm{x}$.

where:

$\mathrm{E}$ and $\mathrm{V}$ is side and node of graph $G$ respectively; ${ }^{K}$ is complete graph in which any two nodes are adjacent.

To solve the problem about thickness calculation of general graph, the author discusses the thickness calculation method for undigraph and proposes calculation formula and detailed algorithm and application instances for calculating thickness $\theta(G)$.

\section{Formula for Calculation of Thickness $\theta(G)$ of Graph $G$}

According to the requirement presented in part I of this paper, which the side derived subgraph shall be conformed, the basic idea on which the author discusses the calculation formula is to seek the minimum division of side derived subgraph of graph $G$ according to the definition of $\theta(G)$, namely, the minimum value of $n$.

On the basis of that mentioned above, two formulas are proposed in this paper:

\subsection{Calculation formula}

Suppose that the minimum divisions of planar subgraph of graph $\mathrm{G}$ by side are $G\left(E_{1}\right), G\left(E_{2}\right), \ldots, G\left(E_{n}\right)$, then

$$
\theta(G)=n
$$

where:

$G\left(E_{1}\right)$ is maximal planar subgraph

$G\left(E_{2}\right)$ is complement graph of $G\left(E_{1}\right)$ relative to $\mathrm{G}$

$G\left(E_{n}\right)$ is complement graph of union set of [ $G\left(E_{1}\right)$ to $G\left(E_{n-1}\right)$ ] relative to $\mathrm{G}$

$\mathrm{n}$ is number of minimum divisions of planar subgraph of graph $\mathrm{G}$ derived by side

So called minimum division of side-derived planar subgraph refers to that $G\left(E_{3}\right)$ can be divided only if $G\left(E_{2}\right)$ is non-planar after $G\left(E_{1}\right)$ is divided; otherwise, the division can not be continued, and so on. 
If $G\left(E_{2}\right)$ is planar, then $\mathrm{n}=2$; if $G\left(E_{2}\right)$ is non-planar, continue to divide and $G\left(E_{3}\right)$ is one of divisions; if $G\left(E_{3}\right)$ is planar, $\mathrm{n}=3, \ldots \ldots$ and so on, and finally minimum division and corresponding value of $\mathrm{n}$ can be determined.

Furthermore, for the divided side derived subgraphs from $G\left(E_{1}\right)_{\text {to }} G\left(E_{n}\right)$, it is necessary to use Euler's formula $v-m+r=2$ to check whether they are planar graphs and satisfy $\sum_{i=1}^{n} E_{i}=E$, so as to check the correctness of division of side derived subgraphs.

\subsection{Formulas}

Divide graph $\mathrm{G}$ into $\mathrm{n}$ side derived subgraphs, then

$$
\begin{gathered}
E=E_{1}+E_{2}+\cdots E_{n} \\
\sum_{i=1}^{n} V_{i}=V_{1}+V_{2}+\cdots V_{n} \\
\sum_{i=1}^{n} F_{I}=F_{1}+F_{2}+\cdots F_{n}
\end{gathered}
$$

where,

E: number of sides of graph $\mathrm{G}, \mathrm{V}_{1}$ to $\mathrm{V}_{\mathrm{n}}$ : nodes of $G\left(E_{1}\right)$ to $G\left(E_{n}\right), \mathrm{F}_{1}$ to $\mathrm{F}_{\mathrm{n}}$ : inner surfaces of $G\left(E_{1}\right)$ to $G\left(E_{n}\right)$.

Because subgraphs from $G\left(E_{1}\right)_{\text {to }} G\left(E_{n}\right)$ are all planar graphs, they all satisfy the satisfy Euler's formula: $V-E+F=2$. On the basis of author's research on Euler's formula, number "2" in it is actually the sum of value of one inner surface (Yin surface) and outer surface(Yang surface), i.e. 1+1=2. In this paper, Let suppose that the numbers of inner surfaces of $G\left(E_{1}\right)$ to $G\left(E_{n}\right)$ are $\mathrm{F}_{1}$ to $\mathrm{F}_{\mathrm{n}}$.

Then, accumulate all Euler's formulas which the subgraphs $G\left(E_{1}\right)$ to $G\left(E_{n}\right)$ are met:

$$
\sum_{i=1}^{n} V_{i}-E+\sum_{1}^{n} F_{i}
$$

is the sum of numbers of outer(Yang) surfaces of $G\left(E_{1}\right)$ to $G\left(E_{n}\right)$. Since each graph from $G\left(E_{1}\right)$ to $G\left(E_{n}\right)$ has an outer (Yang) surface which value is "1" and is just the value of this graph, "1", so, sum of numbers of outer(Yang) surfaces of $G\left(E_{1}\right)$ to $G\left(E_{n}\right)$ just equals to the sum of numbers of graphs from $G\left(E_{1}\right)$ to $G\left(E_{n}\right)$ and the value of $\mathrm{n}$ which is the minimum division.

So, the second formula for calculating $\theta(G)$ is derived:

$$
\theta(G)=\sum_{i=1}^{n} V_{i}-E+\sum_{I=1}^{N} F_{i}
$$




\section{Calculation Instances}

After the text edit has been completed, the paper is ready for the template. Duplicate the template file by using the Save As command, and use the naming convention prescribed by your conference for the name of your paper. In this newly created file, highlight all of the contents and import your prepared text file. You are now ready to style your paper; use the scroll down window on the left of the MS Word Formatting toolbar.

4.1. Calculate the thickness of graph ${ }^{K_{3,3}}$ (figure 1)

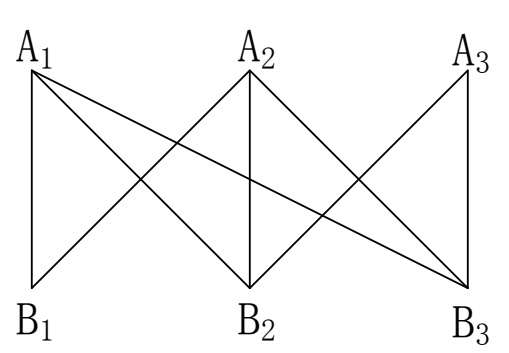

Figure 1. $K_{3,3}$

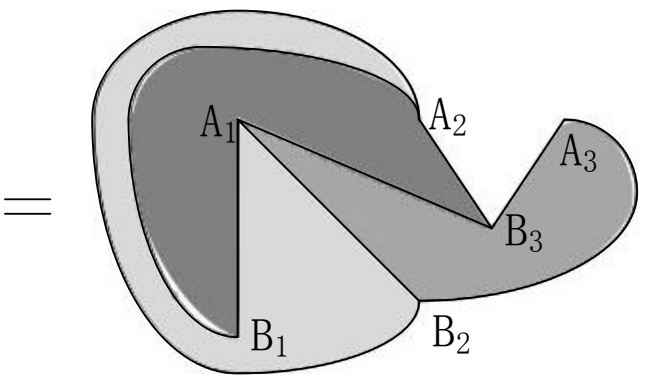

$\mathrm{G}\left(\mathrm{E}_{1}\right)$

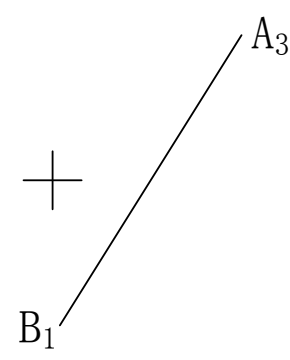

$\mathrm{G}\left(\mathrm{E}_{2}\right)$

Solution:

1) divide $K_{3,3}$ into two side derived subgraphs $G\left(E_{1}\right)$ and $G\left(E_{2}\right)$

2) Check $G\left(E_{1}\right)$ and $G\left(E_{2}\right)$

$G\left(E_{1}\right): V_{1}=6 \quad E_{1}=8 \quad F_{1}=4 \quad V_{1}-E_{1}+F_{1}=2$ satisfy Euler's formula

$G\left(E_{2}\right): V_{2}=2 \quad E_{2}=1 \quad F_{2}=1 \quad V_{2}-E_{2}+F_{2}=2$ satisfy Euler's formula

$K_{3,3}: E=9, G\left(E_{1}\right): E_{1}=8, G\left(E_{2}\right): E_{2}=1$ $K_{3,3}$

So, $E_{1}+E_{2}=8+1=9=E$, sum of sides of side derived subgraphs equals to the number of sides of graph

So, division for side derived subgraphs is correct.

3) Calculate the thickness $\theta(G)$ of graph $K_{3,3}$

According to (1), $\theta(G)=1+1=2$

According to (2),

$\theta(G)=(6+2)-(8+1)+(3+0)=8+9-3=2$

The results from (1) and (2) are equal, then, the thickness of $\theta(G)$ of graph $K_{3,3}$ is 2. 


\subsection{Calculate the thickness of graph $K_{5}$ in figure2}

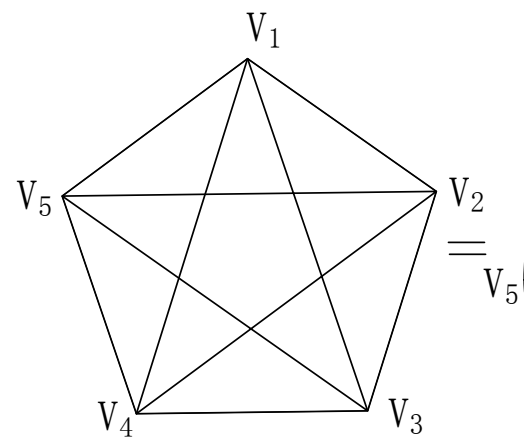

Figure $2 . K_{5}$

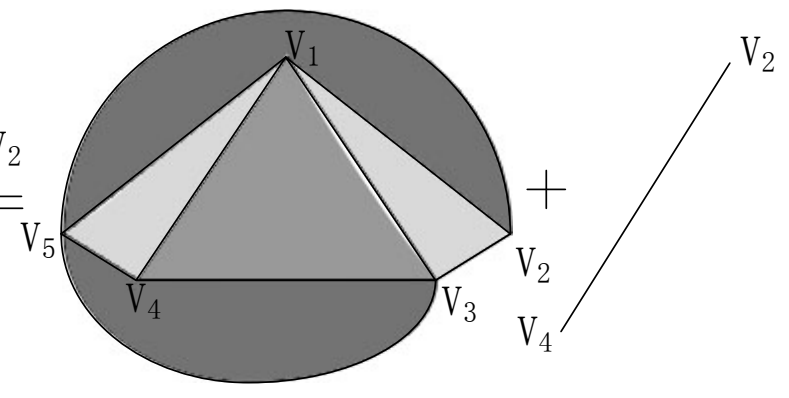

$G\left(E_{1}\right)$

Solution:

1) Divide $K_{5}$ into two side derived subgraphs $G\left(E_{1}\right)$ and $G\left(E_{2}\right)$

2) Check the correctness of the division of side derived subgraphs $G\left(E_{1}\right)$ and $G\left(E_{2}\right)$ of $K_{5}$

$G\left(E_{1}\right): V_{1}=5 \quad E_{1}=9 \quad F_{1}=6 \quad V_{1}-E_{1}+F_{1}=2$ correct

$G\left(E_{2}\right): V_{2}=2 \quad E_{2}=1 \quad F_{2}=1 \quad V_{2}-E_{2}+F_{2}=2$ correct

$K_{5}: E=10 \quad E_{1}=9 \quad E_{2}=1 \quad E_{1}+E_{2}=9+1=10=E$ correct

3) Calculate the thickness $\theta(G)$ of graph $K_{5}$

It is obvious according to (1)

$\theta(G)=1+1=2$

according to (2)

$\theta(G)=(5+2)-10+(5+0)=7-10+5=2$

The results from (1) and (2) are equal, then, the thickness of $\theta(G)$ of graph $\mathrm{K}_{5}$ is 2 .

\subsection{Calculate the thickness of graph show in figure3}

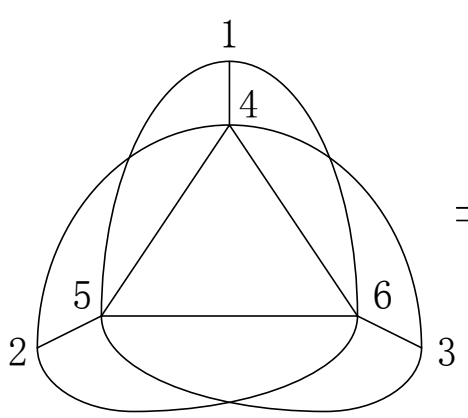

Figure $3 \quad \mathrm{~V}=16$ $\mathrm{E}=12$

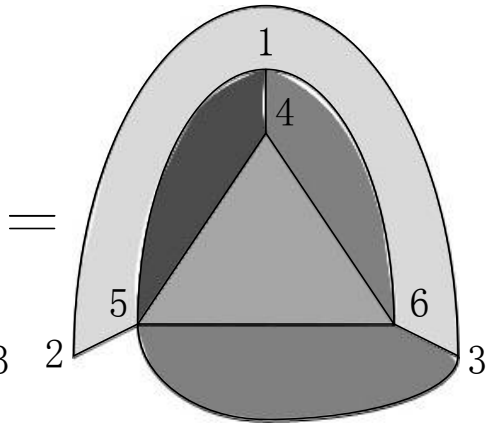

$\mathrm{G}\left(\mathrm{E}_{1}\right)$

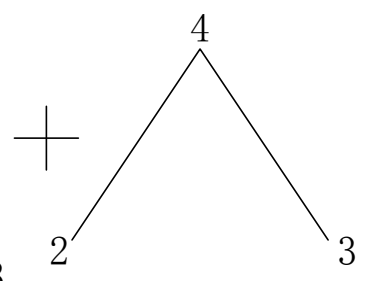

G (E2) 
Solution:

1) Divide the original graph into two side derived subgraphs $G\left(E_{1}\right)$ and $G\left(E_{2}\right)$ and check the correctness of the division

$G\left(E_{1}\right): V_{1}=6 \quad E_{1}=10 \quad F_{1}=6 \quad V_{1}-E_{1}+F_{1}=2$ correct

$G\left(E_{2}\right): V_{2}=3 \quad E_{2}=2 \quad F_{2}=1 \quad V_{2}-E_{2}+F_{2}=2$ correct

$E_{1}+E_{2}=10+2=12=E$ correct

2) Calculate the thickness $\theta(G)$ of this graph

According to (1), $\theta(G)=1+1=2$

According to (2),

$\theta(G)=(6+3)-12+(5+0)=9-12+5=2$

All thicknesses calculated by (1) and (2) are 2 .

4.4. Calculate the thickness of the graph in figure 4

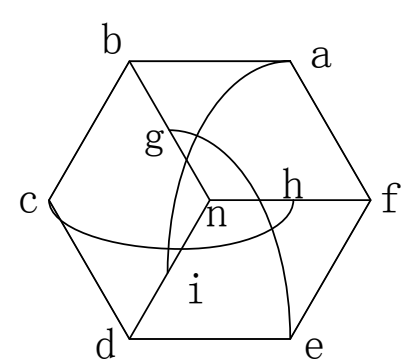

figure4

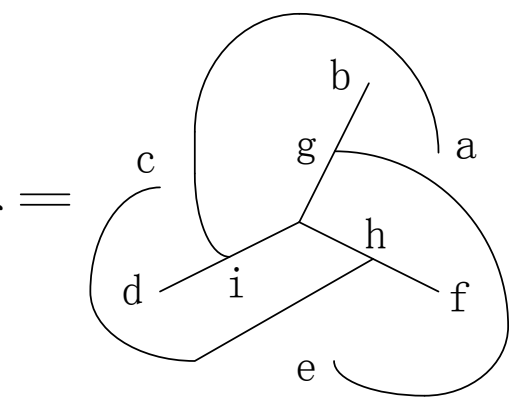

$G\left(E_{1}\right)$

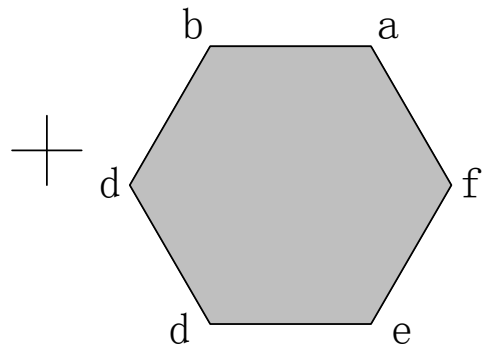

$G\left(E_{1}\right)$

Solution:

1) Divide side derived subgraphs

$G\left(E_{1}\right): \quad E_{1}=9 \quad V_{1}=10 \quad F_{1}=1 \quad V_{1}-E_{1}+F_{1}=2$ So, $G\left(E_{1}\right)$ is planar graph

$G\left(E_{2}\right): \quad E_{2}=6 \quad V_{2}=6 \quad F_{2}=2 \quad V_{2}-E_{2}+F_{2}=2 \quad$ So, $G\left(E_{2}\right)$ is planar graph

In figure $4, E=15$, sum of sides of $G\left(E_{1}\right)$ and $G\left(E_{2}\right)$ is $9+6=15$,so, division for figure 4 is correct.

2) Calculate the thickness of figure 4

According to (1), $\theta(G)=1+1=2$

According to (2),

$\theta(G)=\left(V_{1}+V_{2}\right)-\left(E_{1}+E_{2}\right)+\left(F_{\text {linner }}+\mathrm{F}_{\text {2inner }}\right)$

$=(10+6)-(9+6)+(0+1)=16-15+1=2$

The results from two formulas are equal, so, the thickness of figure 4 is 2 . 


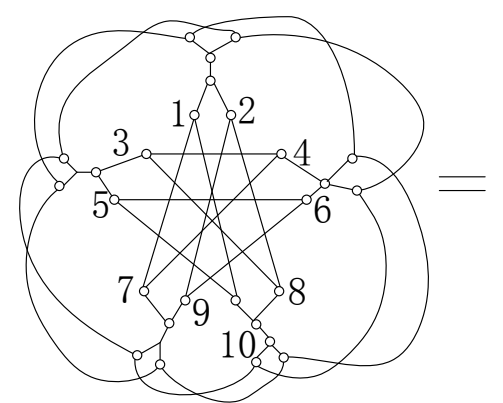

Figure 5
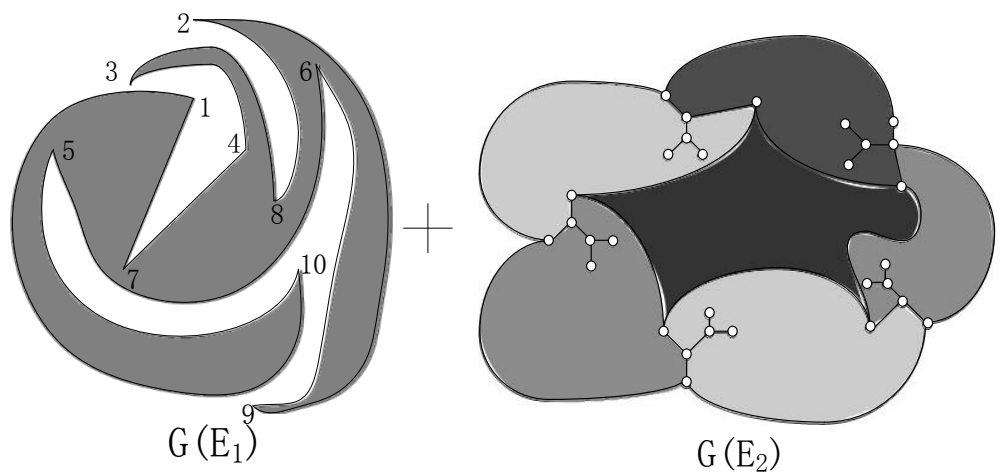

Solution:

1) Divide side derived subgraphs

$G\left(E_{1}\right): V_{1}=10 \quad E_{1}=10 \quad F_{1}=2 \quad V_{1}-E_{1}+F_{1}=2$ So, $G\left(E_{1}\right)$ is planar graph

$G\left(E_{2}\right): V_{2}=30 \quad E_{2}=35 \quad F_{2}=7 \quad V_{2}-E_{2}+F_{2}=2$ So, $G\left(E_{2}\right)$ is planar graph

In figure $5, E=45$, sum of sides of $G\left(E_{1}\right)$ and $G\left(E_{2}\right)$ is $10+35=45$,so, division for figure 6 is correct.

2) Calculate the thickness of figure 5

According to (1), $\theta(G)=1+1=2$

According to (2)

$\theta(G)=\left(V_{1}+V_{2}\right)-\left(E_{1}+E_{2}\right)+\left(F_{\text {linner }}+\mathrm{F}_{2 \text { inner }}\right)$

$=(10+30)-(10+35)+(1+6)=40-45+7=2$

The results from two formulas are equal, so, the thickness of figure 5 is 2 .

\section{Conclusion}

- In fact, (1) and (2) in this paper are all applied to calculate the thickness of graph $\mathrm{G}$ which are derived according to definition of thickness of graph. So, the concept is clear and the calculation is reliable.

- This paper proposes the method for checking the correctness of side derived subgraphs obtained from division of graph $\mathrm{G}$ by using Euler's formula. This method is reasonable and scientific and provides reliability to the calculation method in this paper. So, it will be used conveniently.

- The concept of inner (Yin) surface and outer (Yang) surface has been presented in previous literature of the author [3] which can be referenced for details.

- In the procedure for calculating the thickness $\theta(G)$ of graph $G$, Euler's formula for planar graph $V-E+F=2$ is used to divide side derived subgraphs of $\mathrm{G}$ and check the correctness of the division for these side derived subgraphs. The checking results show that graph $G$ can be considered as one inner surface and one outer surface (infinite surface) before dividing side derived subgraphs and the union of side derived subgraphs still satisfy Euler's formula for planar graph $V-E+F=2$ after dividing. This shows that the number " 2 " is constant for side derived subgraphs divided from graph $G$, i.e. the "surface" of graph $G$ is constant. This can be called the conservativeness of graph. The author ever considered the inner surface and the outer surface as Yin surface and Yang surface respectively. Then the point that graph $G$ conforms to balance of Yin and Yang can be made out according to theory in Book of Changes created in ancient China. 
- The author considers that all algorithms are constant if time and space it used is taken into account and "complexity" is also constant.

- For explaining the conservativeness of graph, figure 4 is taken as an example. Divide the graph in figure 4 into two subgraphs $G\left(E_{1}\right)$ and $G\left(E_{2}\right) . G\left(E_{1}\right)$ has an inner (Yin) surface (abcdef) and an outer (Yang) surface (infinite surface). Union of $G\left(E_{1}\right)$ and $G\left(E_{2}\right)$ still has an inner (Yin) surface and an outer (Yang) surface same as the situation before $G$ is divided into side derived subgraphs. The total number is all 2 , which shows that the surfaces are constant.

- It shall be emphasized that call inner surface as Yin surface and outer surface as Yang surface not only make the number of inner (Yin) surfaces more clear and visual when coloring, but also make the number "2" in Euler's formula for planar graph can be reasonably explained by using Yin and Yang balance theory in Book of Changes. Sum of one "Yin surface" and one "Yang surface" is 2, i.e. $1+1=2$, which is a novel explanation proposed in this paper on number " 2 " in Euler's formula for planar graph. Up to now, there is no relevant explanation on number "2" was found in any other literatures. Point that calls inner and outer surfaces as Yin and Yang surfaces and uses the theory in Book of Changes to explain Euler's formula for planar graph $V-E+F=2$ is a innovation and unique view of author's graph theory knowledge.

If graph $G$ is a non-planar graph, there are still some sides interconnecting each other except for nodes even if any modification is done to graph $G$. it shows that the number of intersection points of sides relate to the thickness of graph. Theoretically, the thickness of graph $G_{\text {ought }}$ to be function of number of intersection points of sides on the basis of qualitative analysis. How to establish this functional relationship is a new issue needed to be studied in the future. If this functional relationship can be established, the thickness of graph $\mathrm{G}$ can be calculated when the number of intersection points of side presented after modification of graph $G$ (planar embedding) is determined. This will form the third formula for calculating thickness $\theta(G)$ of graph $G$.

- The lower bound, i.e. minimum value of thickness of graph $G, \theta(G)$ is 2 . It is unknown whether the upper bound of $\theta(G)$ exists. The author guesses that the upper bound of $\theta(G)$ maybe exist which needs to be deeply research in the future.

\section{References}

[1] Wang Shuhe. Graph theory. Science Press, 2008, pp130-137.

[2] Tian Wencheng, Zhou Luxin. Discrete Mathematics. Tianjin University Press, 2001, pp187-225.

[3] Yan Cui. Chao-Dong Cui , "Research on necessary and sufficient condition for Hamilton graph," 2010 2rd International Conference on Intellectual Technique in Industrial Practice. China, vol. 1, pp.376-380, September 2010.

[4] Ainouche A, Kouider M. Hamiltonism and partially square graphs[J].Graphs and Combinatorics, 1999,15(3):257 265.

[5] Liu,Yiping,Tian,Feng,Wu,Zhengsheng. "Sequence concerning Hamiltonicity of graphs" Journal of Nanjing Normal University(Natural Science),1995,18(1):19 28.

[6] Min Xu "Several New Results on Hamiltonicity of Graphs" Outstanding Dissertation of Chinese text databases 2002,(02)

[7] li-li Zhang "Several Sufficient Conditions on Hamiltonian Properties in Graphs" Outstanding Dissertation of Chinese text databases, 2002,(02)

[8] Jin Yang "Several Sufficient Conditions for Hamiltonian Graphs" Outstanding Dissertation of Chinese text databases, 2003,(02) 